\title{
STEGANOGRAPHY AlgoRITHM IN DIFFERENT COLOUR MODEL USING AN ENERGY ADJUSTMENT APPLIED WITH DISCRETE WAVELET TRANSFORM.
}

\author{
B.E. Carvajal-Gámez ${ }^{1}$, F.J. Gallegos-Funes ${ }^{1}$ and A.J. Rosales-Silva ${ }^{1}$ \\ ${ }^{1}$ National Polytechnic Institute of Mexico, \\ ${ }^{1}$ Mechanical and Electrical Engineering Higher School, \\ México, D. F. \\ becarvajalg@gmail.com, \\ fgallegosflipn.mx, arosaless@ipn.mx
}

\begin{abstract}
When color images are processed in different color model for implementing steganographic algorithms, is important to study the quality of the host and retrieved images, since it is typically used digital filters, visibly reaching deformed images. Using a steganographic algorithm, numerical calculations performed by the computer cause errors and alterations in the test images, so we apply a proposed scaling factor depending on the number of bits of the image to adjust these errors.
\end{abstract}

\section{KEYWORDS}

Color images, color models, steganographic algorithms, scaling factor.

\section{INTRODUCTION}

The information concealment concerns to the process of integrating information or data elements into music, video, and images [1]. The hidden information in steganography does not have relationship with the host image. The information contained in the host image is just a distraction to the receiver, so this is of no so much interest in its full recovery, but the host image must have the minimum quality, considered because any relief, color or misplaced pixels can cause some suspicion and is susceptible to extract the hidden information without authorization from the transmitter. The most important is the hidden information, which must have a full recovery. Steganography in our days performs vital importance since it is a support tool to the copyright protection, which the authentication processes allow the distribution and legal use of different material. Therefore, several steganographic methods have been proposed, which are effective and secure in the transmission or copying of information because these are based on half visibly innocent as a carrier of information and only with a key or password can access to hidden information inserted. This information does not substantially alter the host image, however, is susceptible to Human Vision System (HVS) [2]. The host image with the hidden information is known as stego-image. Typically, there are two techniques to realize a stego-image: in the space domain and frequency domain. The most common method for space domain is the Last Significant Bit (LSB), which is the modification of the least significant bit in each pixel of the image [3]. In the Frequency Domain, the Discrete Fourier Transform (DFT), the Discrete Cosine Transform (DCT), and Discrete Wavelet Transform (DWT) are used to transform the spatial pixel values in frequency coefficients [4]. A number of factors cause in an edge some sensitivity to the 
Signal \& Image Processing : An International Journal (SIPIJ) Vol.3, No.4, August 2012

human eye and the noise effects of noise in the human eye as the luminance, frequency band, and texture. The human eye is less sensitive to noise in high frequency sub band [5]. The sensitivity to noise of the human eye in the textures that conforms an image is less if is closer to the edges. Based on these observations, an adaptable model for image compression using wave coefficients quantization was developed [5]. A method was developed using a concealment function to compute the weight factors into the pseudo random binary sequence in the high frequency components of the host image [5]. In this paper, we use the steganographic method implemented in [6] with some modifications. A scaling factor depending directly on the number of bits of the host image is proposed to ensure that with the use of steganographic technique does not affect the host image and the hidden information is not visible to the HVS. Applying the scaling factor the energy generated by the host image is preserved to approximate the original image, eliminating any visual disturbance, being imperceptible to the human eye.

\section{Proposed METHOD}

Proposed steganographic algorithm is based on the DWT [6, 7]. The image resolution applying DWT is divided into 4 frequency sub bands called LL, LH, HL, and HH, also known as sub matrix a, sub matrix h, sub matrix $v$ and sub matrix $d$ see Figure 1.

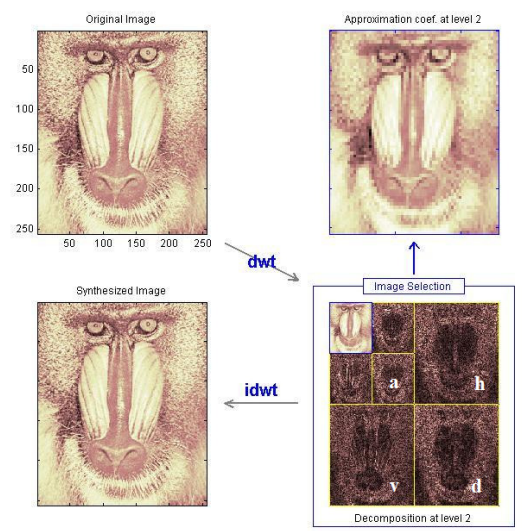

Figure1. Two levels decomposition using DWT in the Mandrill image.

Each subband is a copy of the original image but in different frequency level which provides a certain amount of energy [8, 9]. The proposed steganographic algorithm based on wavelet's theory is shown in Figure 2 [7]. The second block of Figure 2 refers to the double decomposition wavelet applied to the hide image and a simple decomposition wavelet to the host image. Having obtained 4 subbands LL, LH, HL, and HH, we choose the subband LH to perform the hiding,that is because it contains the edges of the image, known as wavelet components of low-high frequencies. This election is because the edges are considered natural noisy areas which are suitable to hide. Later for the hidden image there is elected the subband LL which consists of the components wavelets which contribute $90 \%$ of entire energy of the image. The third block refers to obtaining the standard deviation $\sigma_{R}$, with it to apply the following criterion of insertion: $\sigma_{R} \leq x[n / 2, m / 2]$ then $y\lfloor n, m\rfloor$, where $x|n, m|$ is the host image and $y\lfloor n, m\rfloor$ (see Figure 2) is the hidden image. Finally, we perform the Inverse Discrete Wavelet Transform to obtain the modified image (with the host image inserted the hide image). 
Signal \& Image Processing : An International Journal (SIPIJ) Vol.3, No.4, August 2012

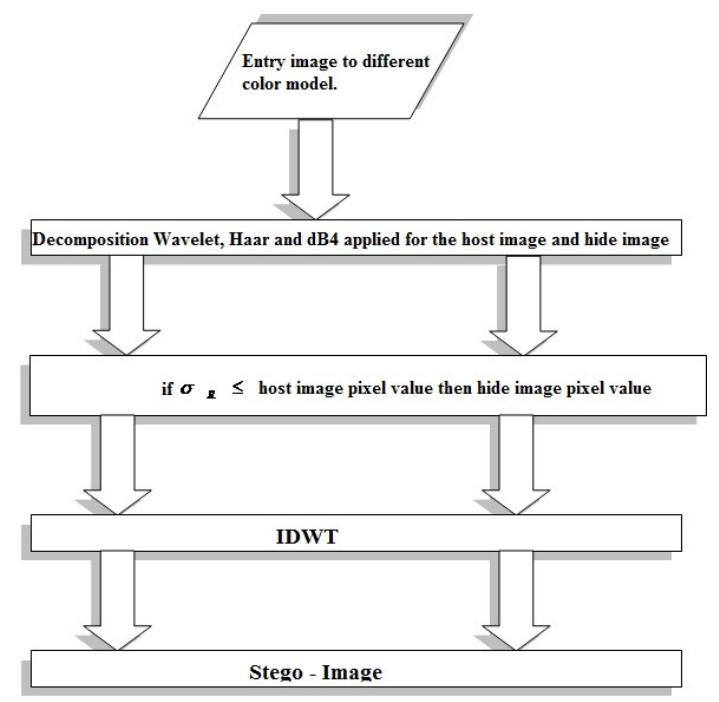

Figure 2. Block diagram of the steganographic algorithm.

DWT is closely linked to the multi-resolution analysis, that is, the observation of the signal or the image at different frequencies [10], which allows a broader knowledge of the signal and facilitates the fast computation when the wavelet family is orthogonal $[6,11,12,13]$. The wavelets $\Psi$ can be obtained such that the family moved for $j$ and dilated for $n$,

$$
\psi_{j, n}(t)=\frac{1}{\sqrt{2^{j}}} \psi \frac{t 2^{j} n}{2^{j}}{ }_{j, n \in Z^{2}}
$$

it is a orthonormal base of $L^{2}(R)[6,11,12,13]$. These orthogonal wavelets transport information about the changes of the signal to the resolution $2^{-j}$. Then, the analysis of multi-resolution appears: an image will be modeled with orthogonal projections on vector space of different resolution, $P V_{j} f, V_{j} \subset L^{2}(R)[12,13]$. The quantity of information in every projection will depend on the size of the orthogonal projection $V_{j}$. The multi-resolution analysis calculates the approach of signals to different resolutions with orthogonal projections in spaces $\left\{V_{j}\right\}$. Also, the multi-resolution approaches are characterized completely by a particular discrete filter that controls the loss of information along the different resolutions. The approach of a function $f$ with a resolution $2^{-j}$ comes specified by a discrete sampling grid, which provides local averages of $f$ in a neighborhood of proportional size to $2^{j}$. This means that the approach of a function to a resolution $2^{-j}$ is defined as an orthogonal projection in a space $V_{j} \subset L^{2}(R)$ [13]. The space $V_{j}$ regroups all the possible approaches to the resolution $2^{-j}$. We consider, in this study, that the resolution $2^{-j}$ corresponds with a scale 2 . The function $f(t)$ can be reconstructed from the discrete wavelets coefficients $W_{f}(j, n)$ in the following way,

$$
f(t)=A \sum_{j} \sum_{n} W_{f}(j, n) \psi_{j, n}(t)
$$

where $j$ is the scale factor, $n$ is the movement factor and $\mathrm{A}$ is a constant that no depends on $f(t)$. Scale factor $j>0$ is always used. The wavelet is dilated when the scale $j>1$, and it is contracted 
Signal \& Image Processing : An International Journal (SIPIJ) Vol.3, No.4, August 2012

when $j<1$. This way, changing the value of $j$ the different range from frequencies is covered. Big values of the parameter $j$ correspond to frequencies of minor range, or a big scale of $\psi_{j, n}(t)$ small values of $j$ correspond to frequencies of minor range or a very small scale of $\psi_{j, n}(t)[14,15]$. Finally, the signal $f(t)$ can be compressed or expand in the time. This will have little certainly after effects in the plane of frequencies [15],

$$
\begin{aligned}
& f(t) \text { compression by a factor } 2^{j}(s) f_{s}(t)=\frac{1}{\sqrt{2^{j}}} f \frac{t}{s} \\
& \hat{f}(w) \text { compression by a factor } \frac{1}{2^{j}} \hat{f}_{2^{j}}(w)=\frac{1}{\sqrt{2^{j}}} 2^{j} \hat{f}\left(2^{j} w\right)=\sqrt{2^{j}} \hat{f}\left(2^{j} w\right)
\end{aligned}
$$

where $\hat{f}(w)$ represent the reconstruction of $f(t)$.

The coefficient of the decomposition of a function $f$ in an orthogonal base of wavelets is calculated by a subsequent algorithm of discrete convolution of $h$ and $g$, and realizes a sampling of the eq. (4) and (5),

$$
\begin{aligned}
& x_{\text {low }}[k]=\sum_{n} x[n, m] h[2 k-l], \\
& x_{\text {high }}[k]=\sum_{n} x[n, m]_{g}[2 k-h],
\end{aligned}
$$

where $x[n, m]$ is an image, $x_{\text {low }}[k]$ and $x_{\text {high }}[k]$ are outputs of the Low Pass Filter (LPF) and High Pass Filter HPF respectively; $g[2 k-l]$ and $h[2 k-h]$ represent the impulse response of LPF and HPF, respectively, sub-sampled by a factor of 2 as expressed in eq. (4) and (5) [10]. These coefficients $x_{\text {low }}[k]$ and $x_{h i g h}[k]$ are calculated by cascades of discrete filters, through of convolution and sampling. In the case of images, the host image $x[n, m]$ must pass through a series of mirror filters banks in quadrature $[9,10,11]$. The filter $h$ removes the components high-high and high-low frequencies of the $x[n, m]$, while $g$ removes the components low-low and low-high frequencies. For the reconstruction, an interpolation is realized, inserting zeros and expanding $x_{\text {high,high }}, x_{\text {high,low }}$, $x_{\text {low, high }}, x_{\text {low,low. }}$. This filtering and decimation process in a continuous way is known as subband coding. Figure 3 depicts the decomposition of the discrete wavelet for a RGB color image, it is interpreted as the decomposition of the submatrix R (representing the Red channel) represented by $X_{R}[n, m]$, for the first $X_{R}[n, m]$ decomposition shown in Figure 3 applies to itself step of the low-pass filter through the rows and columns to obtain subband LL to previously mentioned of the image, in the second decomposition is applied the low-pass filtering through the rows and columns obtain the subband LH, the third decomposition is similar to HL but reverses the first filter is the high-pass filter and later the low-pass filter and get the sub matrix $\mathbf{v}$ and finally for subband $\mathrm{HH}$ filtering is applied throught in rows and columns with the high-pass filter. The terms $h[m]$ or $h[n]$ and $g[m]$ or $g[n]$ represent the impulse response of the LPF and HPF respectively, $x h$ and $x g$ represent each subband o sub images obtained that are decimated by a factor of 2 . 
Signal \& Image Processing : An International Journal (SIPIJ) Vol.3, No.4, August 2012

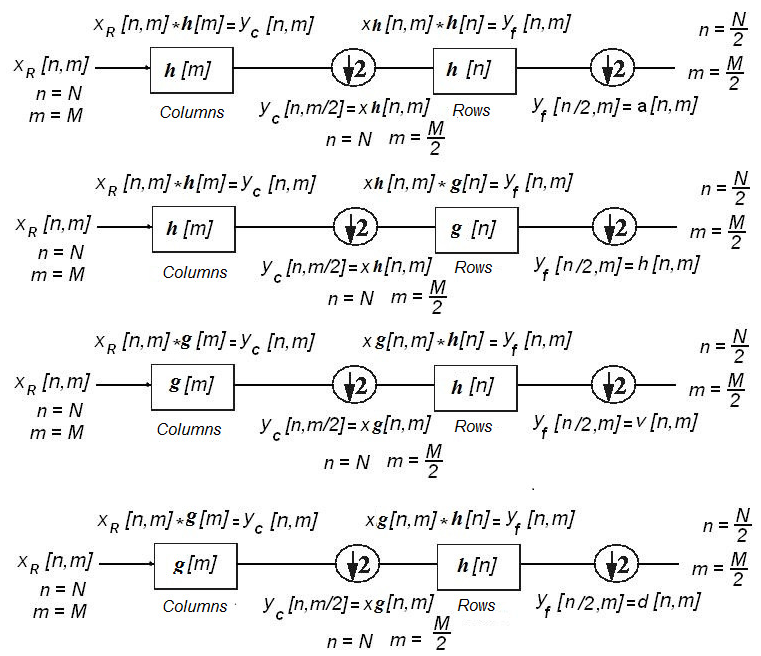

Figure 3. Filter banks for encoding sub-bands representing the discrete wavelet decomposition of an image.

The discrete wavelet reconstruction can be computed by an inverse of the procedure of decomposition beginning for the level of resolution lower in the hierarchy and working from right hand to left side with the diagram of the Figure 3. The most of applications of steganographic algorithms are given in grayscale images, and the energy conservation in the wavelet domain is related to the factor $1 / \sqrt{2}[9,15]$. In applying the proposed steganographic algorithm to the subband LH is necessary to use a scaling factor that works with a 24-bit RGB color images or $\mathrm{YcbCr}$ or Hue, Value, Saturation(HSV) color model, this scaling factor is closely related to energy conservation applied in the theory of wavelets. However, in the RGB color images we propose the following scaling factor,

$$
1 / \sqrt{ } 2^{n},
$$

where $n$ is directly dependent on the number of bits that integrate the image.

\section{EXPERIMENTAL RESULTS}

In the optimization and evaluation of algorithms in digital image processing, the peak signal to noise relation (PSNR) is the criterion most frequently used to evaluate the quality of images [16]. However, the use of image qualitative measures is based on the properties of HVS, the models usually are embedded into HVS sensitivity to light and spatial frequency [17]. Many measures used in the images during the visual information processing belong to the group of measures of difference in distortion [7], is base on the difference between the original and modified images. The most common distortion measure is the PSNR defined as,

$$
\mathrm{PSNR}=10 \log \frac{(255)^{2}}{\mathrm{MSE}}, \mathrm{dB}
$$

where, $M S E=\frac{1}{M_{1} M_{2} M_{3}} \sum_{k=1}^{M_{1} M_{2} \sum_{i} M_{3}}\|y(k, i, j)-x(k, i, j)\|_{L_{2}}^{2}$ is the Mean Square Error, $M_{1}, M_{2}$ and $M_{3}$ are the image dimensions, $y(k, i, j)$ is the $3 \mathrm{D}$ vector value of the pixel in the $(k, i, j)$ location of the stego-image, $x(k, i, j)$ is the corresponding pixel in the original host image, and $\|\cdot\|_{L_{2}}$ is the 
$\mathrm{L}_{2}$-vector norm. The Normalized Color Deviation (NCD) is used for the quantification of the color perceptual error,

$$
N C D=\frac{\sum_{i=1}^{M_{1} M_{2}}\left\|\Delta E_{L u v}(i, j)\right\|_{L_{2}}}{\sum_{i=1}^{M_{1} M_{2}} \sum_{j=1}\left\|E_{L u v}^{*}(i, j)\right\|_{L_{2}}}
$$

Here, $\left\|\Delta E_{L u v}(i, j)\right\|_{L_{2}}=\left(\Delta L^{*}(i, j)\right)^{2}+\left(\Delta u^{*}\right)^{2}+\left(\Delta v^{*}\right)^{2}{ }^{1 / 2}$ is the norm of color error; $\Delta L^{*}, \Delta u^{*}$, and $\Delta v^{*}$ are the difference in the $L^{*}, u^{*}$, and $v^{*}$ components, between the two color vectors that present the stego and host images for each pixel $(i, j)$ of an image, and $\left\|E_{L u v}^{*}(i, j)\right\|_{L_{2}}=\left(L^{*}\right)^{2}+\left(u^{*}\right)^{2}+\left(v^{*}\right)^{2} \quad 1 / 2$ is the norm of the host image pixel vector in the $L^{*} u^{*} v^{*}$ space.

The quality index $(\mathrm{Q})$ is provided to demonstrate the quality of the stego-images [2], where $x$ and $y$ are the mean values of the host and stego- images respectively, $\sigma_{x}^{2}$ and $\sigma_{y}^{2}$ are the variances of the host and stego-images respectively, and $\sigma_{x y}=\frac{1}{N \quad \sum} \sum_{i}^{N}\left(x_{i} \quad x\right)\left(y_{i} \quad y\right)$ is the coefficient correlation between host image $x$ and hide image $y$.

$$
Q=\frac{4 \sigma_{x y} \bar{x} y}{\left(\sigma_{x}^{2}+\sigma_{y}^{2}\right)\left(x^{2}+y^{2}\right)}
$$

The Hiding capacity (HC) is computed as follows [18],

$$
H C=M S E^{*} \frac{\text { number of samples in embedding band }}{\text { number of bits of secure data }}
$$

where $H C$ dictates that the number of bits inserted in the host image. Experimental results were conducted with 24-bit RGB images to show our mentioned scaling factor. As mentioned previously, the filtering can distort the images, as will be shown in subsequent tests applied filters to distort the host image with the DWT. An improvement in visual images can be perceived using the proposed scaling scheme. Additionally, We incorporate in the proposed scheme other color spaces such as YCbCr (Luminance, Chromatic blue, Chromatic red), and HSV (Hue, Saturation, Value) to ensure that the visual artifacts appeared in the stego-image are imperceptible, and the difference between the cover and stego image is indistinguishable by the HVS by using the proposed scaling factor. Table 1 shows the performance results in terms of PSNR, MAE (Mean Absolute Error), COI (Correlation), Q, NCD, HC, and RMS (Root Mean Square) in the case of different $n$ values in the scaling factor by using the 320 x 320 RGB color images "Mandrill" as host image and "Lena" as hide image. From Table 1, we can see that the $n$ value increases, the performance results increase too, but the $\mathrm{HC}$ decreases because the hide image is inserted in each value of $n$ in areas that do not affect the clarity of host image. Figure 4 depicts the processed images for stego-image Mandrill (Fig. 4a), 4b), and 4c)) and retrieved secret image Lena (Fig. 4d), 4e), and 4f)) according with Table 1. We observe from this Figure that the best results are obtained when $n=9$, where $n$ represents the bits resolution of the image to hide. From Figs. 4d), 
Signal \& Image Processing : An International Journal (SIPIJ) Vol.3, No.4, August 2012

$4 \mathrm{e}$ ), and $4 \mathrm{f}$ ) one can see that when the value of proposed scaling factor increases as well as the subjective quality of images increases too. Tables 2 and 3 present the simulation results of the same test of Table 1 in the case of use of $\mathrm{YCbCr}$ and $\mathrm{HSV}$ color images [19].

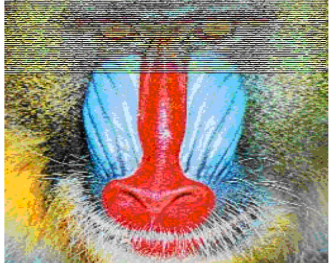

a)

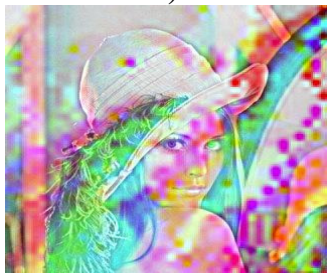

d)

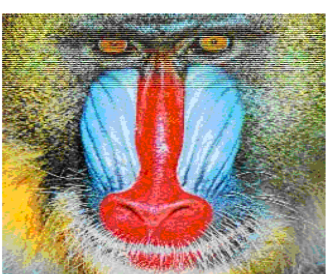

b)

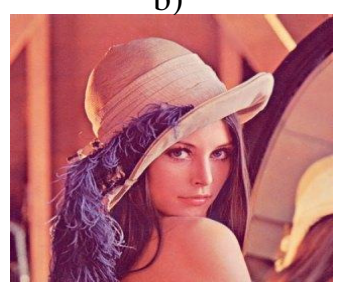

e)

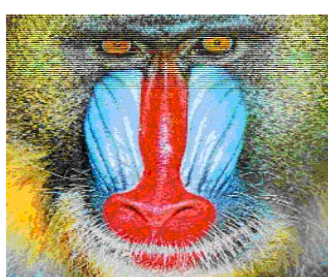

c)

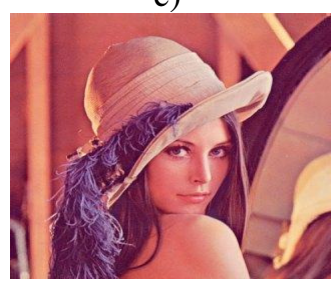

f)

Figure 4. Visual results for different $n$ values in the scaling factor, a) and d) with $n=2, \mathrm{~b}$ ) and e) with $n=5$, and c) and f) $n=9$.

From the simulation results of Tables 1, 2, and 3 we can conclude that as the factor of scale is increasing we see in the results a visual improvement given for Q, COI, PSNR, RMS, etc; but according to this factor of scale is on the increase, a decrease exists in the capacity of insertion, is possible to see that the color model with major sacrifice in capacity of insertion is the HSV that offers the best visual results. From the simulation results of Tables 1, 2, and 3 we can conclude that as the factor of scale is increasing we see in the results a visual improvement given for Q, COI, PSNR, RMS, etc; but according to this factor of scale is on the increase, a decrease exists in the capacity of insertion, is possible to see that the color model with major sacrifice in capacity of insertion is the HSV that offers the best visual results. Table 4, shows the performance results in the $n=10$ case in the scaling factor. Figure 6 presents the visual results according with Table 4 . We also present the error images. The proposed scaling factor $1 / \sqrt{2^{n}}$ for each test presents a different result as can be seen in the previous tests, the scaling factor does not affect the steganographic algorithm preserving the energy of images. It can be seen that in the Lena error image of the Figure 5d), the difference value between the host image and the recovered image is approximately zero providing that the hidden information is almost imperceptible. Finally, depending on the amount of information than we want to hide we can choose the model that best to hide information if that this one deform visibly the host image, in Table 4 we can see than the model of color HSV offers better quality in the stego-image in contrast to the RGB and YCbCr models, nevertheless capacity of insertion gets lost. It is possible to observe that the model RGB offers good results and capacity of insertion does not sacrifice itself. 
Signal \& Image Processing : An International Journal (SIPIJ) Vol.3, No.4, August 2012

Table 1. Performance results for different $\mathrm{n}$ values for RGB color model.

\begin{tabular}{llccccccc}
\hline$N$ & & PSNR db & COI $\%$ & NCD & Q & MAE & HC & RMS \\
\hline \multirow{2}{*}{$n=0$} & Host image & 31.5084 & 0.7852 & 0.0020 & 0.7836 & 10.4878 & $73.06 \mathrm{~Kb}$ \\
& Hide image & 16.5327 & 0.388 & 0.4748 & 0.3586 & 4.9403 & 0.4760 \\
\multirow{2}{*}{$n=2$} & Host image & 31.4999 & 0.8046 & 0.0020 & 0.8040 & 9.9490 & $66.31 \mathrm{~Kb}$ \\
& Hide image & 16.9537 & 0.3889 & 0.4749 & 0.3587 & 4.9471 & & 0.4748 \\
\multirow{2}{*}{$n=5$} & Host image & 36.1233 & 0.9781 & 0.0020 & 0.9792 & 3.2086 & $61.97 \mathrm{~Kb}$ \\
& Hide image & 27.2474 & 0.9985 & 0.0020 & 0.9962 & 2.7714 & 0.4614 \\
\multirow{2}{*}{$n=9$} & Host image & 36.1233 & 0.9908 & 0.0008 & 0.9913 & 2.0309 & $3.835 \mathrm{~Kb}$ & 0.4610 \\
& Hide image & 31.0781 & 0.9980 & 0.0020 & 0.9962 & 2.7714 & & \\
\hline
\end{tabular}

Table 2. Performance results for different $n$ values for $\mathrm{YCbCr}$ color model.

\begin{tabular}{|c|c|c|c|c|c|c|c|c|}
\hline$N$ & & PSNR db & $\mathrm{COI} \%$ & $\mathrm{NCD}$ & Q & $\mathrm{MAE}$ & $\mathrm{HC}$ & RMS \\
\hline \multirow{2}{*}{$n=0$} & Host image & 19.5146 & 0.8713 & 0.0020 & 0.8751 & 7.1762 & $26.34 \mathrm{~Kb}$ & 0.3961 \\
\hline & Hide image & 2.7975 & 0.2008 & 0.0020 & 0.0601 & 178.1406 & & \\
\hline \multirow{2}{*}{$n=2$} & Host image & 19.7324 & 0.8782 & 0.0020 & 0.8816 & 7.0325 & $24.66 \mathrm{~Kb}$ & 0.4694 \\
\hline & Hide image & 36.0827 & 0.9954 & 0.0020 & 0.9962 & 2.7948 & & \\
\hline \multirow[b]{2}{*}{$n=5$} & Host image & 22.1046 & 0.9318 & 0.0015 & 0.9323 & 5.5328 & $12.30 \mathrm{~Kb}$ & 0.4202 \\
\hline & Hide image & 36.0827 & 0.9954 & 0.0020 & 0.9962 & 2.7948 & & \\
\hline \multirow[b]{2}{*}{$n=9$} & Host image & 28.5553 & 0.9836 & 0.0015 & 0.9843 & 2.7372 & $3.339 \mathrm{~Kb}$ & 0.4132 \\
\hline & Hide image & 36.0827 & 0.9954 & 0.0020 & 0.9962 & 2.7948 & & \\
\hline
\end{tabular}

Table 3. Performance results for different $n$ values for HSV color model.

\begin{tabular}{llccccccc}
\hline$N$ & & PSNR db & COI $\%$ & NCD & Q & MAE & HC & RMS \\
\hline \multirow{2}{*}{$n=0$} & Host image & 6.2955 & 0.5217 & 0.0020 & 0.0863 & 113.9768 & $0.359 \mathrm{~Kb}$ & 0.0020 \\
& Hide image & 2.8061 & 0.0592 & 0.0020 & 0.0003 & 177.9792 & & \\
\hline \multirow{2}{*}{$n=2$} & Host image & 20.1709 & 0.9015 & 0.0019 & 0.9067 & 5.6167 & $0.257 \mathrm{~Kb}$ & 0.0017 \\
& Hide image & 36.1233 & 0.9955 & 0.0020 & 0.9962 & 2.7714 & & \\
\hline \multirow{2}{*}{$n=5$} & Host image & 23.5030 & 0.9441 & 0.0010 & 0.9469 & 3.4113 & $0.568 \mathrm{~Kb}$ & 0.0013 \\
& Hide image & 36.1233 & 0.9955 & 0.0020 & 0.9962 & 2.7714 & & \\
\hline \multirow{2}{*}{$n=9$} & Host image & 38.8068 & 0.9985 & 0.0005 & 0.9985 & 0.8092 & $0.0713 \mathrm{~Kb}$ & 0.0012 \\
& Hide image & 36.1233 & 0.9955 & 0.0020 & 0.9962 & 2.7714 & & \\
\hline
\end{tabular}




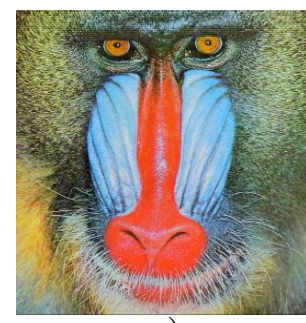

a)

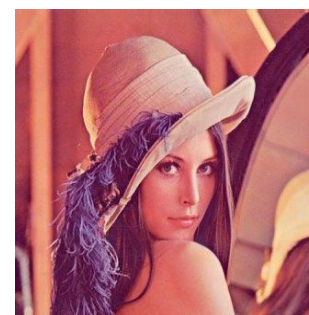

c)

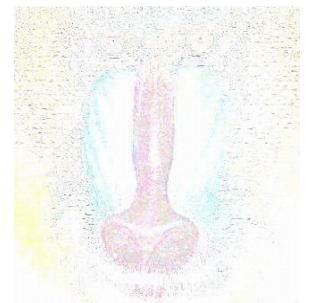

b)

d)

Figure 5. Visual results in the case of $n=10$, a) stego-image "Mandrill, b) error stego-image "Mandrill, c) hide image "Lena", d) error hide image "Lena".

Table 4. Performance results in different color models for $n=10$ in the scaling factor.

\begin{tabular}{|c|c|}
\hline \multicolumn{2}{|c|}{ RGB color model } \\
\hline Host image "Mandrill" & Hide image "Lena" \\
\hline $\mathrm{Q}=0.9934$ & $\mathrm{Q}=0.9962$ \\
\hline $\mathrm{PSNR}=36.1233 \mathrm{~dB}$ & $\mathrm{PSNR}=32.5167 \mathrm{~dB}$ \\
\hline $\mathrm{COI}=99.34 \%$ & $\mathrm{COI}=99.55 \%$ \\
\hline $\mathrm{NCD}=6.0486 \mathrm{e}-4$ & $\mathrm{NCD}=0.0020$ \\
\hline MAE $=1.7022$ & MAE $=2.7714$ \\
\hline $\mathrm{HC}$ & $25.65 \mathrm{~Kb}$ \\
\hline \multicolumn{2}{|c|}{ YCbCr color model } \\
\hline Host image "Mandrill" & Hide image "Lena" \\
\hline $\mathrm{Q}=0.9888$ & $\mathrm{Q}=0.9962$ \\
\hline $\mathrm{PSNR}=30.3913 \mathrm{~dB}$ & $\mathrm{PSNR}=36.0827 \mathrm{~dB}$ \\
\hline $\mathrm{COI}=98.92 \%$ & $\mathrm{COI}=99.54 \%$ \\
\hline $\mathrm{NCD}=9.5940 \mathrm{e}-4$ & $\mathrm{NCD}=0.0020$ \\
\hline $\mathrm{MAE}=2.2044$ & $\mathrm{MAE}=2.7948$ \\
\hline $\mathrm{HC}$ & $2.18 \mathrm{~Kb}$ \\
\hline \multicolumn{2}{|c|}{ HSV color model } \\
\hline Host image "Mandrill" & Hide image "Lena" \\
\hline $\mathrm{Q}=0.9993$ & $\mathrm{Q}=0.9962$ \\
\hline $\mathrm{PSNR}=41.3900 \mathrm{~dB}$ & $\mathrm{PSNR}=36.1233 \mathrm{~dB}$ \\
\hline $\mathrm{COI}=99.92 \%$ & $\mathrm{COI}=99.55 \%$ \\
\hline $\mathrm{NCD}=2.8906 \mathrm{e}-4$ & $\mathrm{NCD}=0.0020$ \\
\hline MAE $=0.6401$ & $\mathrm{MAE}=2.7714$ \\
\hline $\mathrm{HC}$ & $0.068 \mathrm{~Kb}$ \\
\hline
\end{tabular}

\section{CONCLUSIONS}

The RGB, HSV and YCbCr color model images are altered in their energy contribution in each sub-matrix of wavelet decomposition when the steganographic algorithm is applied. It is known that the value of $1 / \sqrt{2}$ is the key factor in the adjustment of the wavelets energy, this adjustment value has been applied only in gray scale images tests. However, the energy conservation factor mentioned above is not valid for true color images, such as formed by 3 submatrices (RGB, HSV 
Signal \& Image Processing : An International Journal (SIPIJ) Vol.3, No.4, August 2012

and $\mathrm{YCbCr}$ ) which separately provides some level of energy, that in sum contributes the quantity of entire energy, because if it is not right or there are energy gap in the components, the images may have a poor display as distortion. By applying the proposed scaling factor $1 / \sqrt{2^{n}}$, there is an adjustment factor for the energy input in each submatrix. It is also noted that when is changing the value of $n$, it adjusts the sharpness and image clarity providing a visible improvement of the visual image. We conclude that the HSV model is suitable for small images. And for larger images it is advisable to use RGB model. The $\mathrm{YCbCr}$ model is the least suitable for steganography.

\section{ACKNOWLEDGMENTS}

This work is supported by National Polytechnic Institute of Mexico and Conacyt.

\section{REFERENCES}

[1] Chia-Chen, L., Wei-Liang, T., Chin-Chen C.: Multilevel reversible data hiding based on histogram modification of difference. Pattern Recognition. 3582- 3591 (2008)

[2] Wang - Zhou, Bovik C. Alan.: A universal quality index. IEEE Signal Processing Letters.81-84 (2002)

[3] Chung-Ming, W., Nan-I W., Chwei-Shyong, T., Min Shiang H.: A high quality steganographic method with pixel value differencing and modulus function. Journal of System and Software. 150-158 (2008)

[4] Yuan-Hui,Y., Chin-Chen, C., Yu-Chen, H.: Hiding secret data in images via predictive coding. Pattern Recognition. 691-705 (2005)

[5] Reddy, A.A., Chatterji, B.N.: A new wavelet based logo-watermarking scheme. Pattern Recognition Lett. 1019-1027 (2005)

[6] Carvajal-Gámez, B. E., Acevedo M., López-Bonilla, J.: Energy conservation in the concealment of information in a video by means of discrete wavelet transform. IeJEMTA 4, 27-31 (2008)

[7] Kutter, M., Petitcolas, F.A.P.: A fair benchmark for image watermarking systems. Proc. SPIE Electronic Imaging '99. Security and Watermarking of Multimedia Contents. 1-14 (1999)

[8] Moon, H.S., You, T., Sohn, M.H., Kim, H.S., Jang, D.S.: Expert system for low frequency adaptive image watermarking: Using psychological experiments on human image perception. Expert Systems with Applications. 674-686 (2007)

[9] Walker, J.: A primer on wavelets and their scientific applications, (2003), Chapman \& Hall/CRC, ISBN: 1584887451, London.

[10] Vetterli, M., Kočević, J.: Wavelets and Subband Coding, (1995), Prentice-Hall, ISBN:9780130970800 New Jersey

[11] Petrosian, A.A., Meyers, F.G.: Wavelets in Signal and Image Analysis, (2002), Kluwer Academic Pub., ISBN:978-1- 4020-0053-9, Berlin.

[12] Debnath, L.: Wavelets and Signal Processing, (2002), Birkhauser, ISBN: 0-8176-4235-8, Berlin.

[13] Vetterli Martin, Kovacevic Jelena and Goyal Vivek K: Fourier and wavelet signal processing, (2011), Alpha version book 2.0. http://fourierandwavelets.org.(2012) 
Signal \& Image Processing : An International Journal (SIPIJ) Vol.3, No.4, August 2012

[14] Y.Sheng, "The Transforms and Applications Handbook". Ed. 2nd, CRC Press, (2000), ISBN: 9780849385957.

[15] Bogges Albert, Narcovich Francis, "A first course in wavelets with Fourier Analysis". Second edition. Ed. Wiley. ISBN:978-0-470-43117-7.

[16] Huanga, K.Q., Wub, Z.Y., Fungc, G.S.K., Chan, H.Y.: Color image denoising with wavelet thresholding based on human visual system model. Signal Processing: Image communication. 115127 (2005)

[17] Ginesu, G., Massidda, F., Giusto, D.D.: A multi-factors approach for image quality assessment based on human visual system model. Signal Processing: Image communication. 316-333(2006)

[18] Sos S. Agaian, David Akopian and Sunil A. D’Souza "Frequency Domain Based Secure Digital Audio Steganography Algorithms," IEEE SP/CAS, 2005 International Workshop on Spectral Methods and Multirate Signal Processing, SMMS, June 20-22, 2005, Riga, Latvia

[19] Data Base Images of the University of Southern of California, (2012), Signal and Image Processing Institute. USC VITTERBI. http://sipi.usc.edu/database/database.php.(2012) 